\title{
ASPECTOS MOTIVACIONALES DE DONANTES VOLUNTARIOS DE SANGRE EN UN MÓVIL DE RECOLECCIÓN DE LA CIUDAD DE BOGOTÁ, COLOMBIA
}

\section{MOTIVATIONAL ASPECTS OF VOLUNTARY BLOOD DONORS IN A COLLECTION POINT OF THE CITY OF BOGOTA, COLOMBIA}

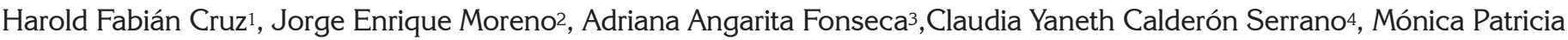 \\ Restrepo Sierra ${ }^{5}$
}

1 Enfermero, Especialización en Estadística Aplicada. Director de Investigación. Fundación Hematológica Colombia FUHECO. Bogotá - Colombia. harolduelbosque@yahoo.es - investigacion@fuheco.org.co 2 Magíster en Ciencias de la Actividad Física. Docente de la Facultad de Enfermería y Rehabilitación. Universidad de la Sabana. Chía - Colombia. jorge.moreno2@ unisabana.edu.co ${ }^{3}$ Magíster en Epidemiología. Universidad de Santander, Bucaramanga - Colombia. adriangarita@udes. edu.co ${ }^{4}$ Bacterióloga, Supervisora Nacional de Reclutamiento. Fundación Hematológica Colombia. Bogotá - Colombia. ccalderon@fuheco.org.co ${ }^{5}$ Comunicadora Social, Subdirectora nacional de promoción de la donación. Fundación Hematológica Colombia. Bogotá - Colombia. mrestrepo@fuheco.org.co

Rev. U.D.C.A Act. \& Div. Cient. 15(1): 19 - 26, 2012

\section{RESUMEN}

La demanda de sangre hace que la donación sea una actividad constante, por lo cual, conocer la motivación para realizarla, se convierte en determinante para la formulación de estrategias que aumenten los donantes y fortalezcan los programas de la donación. El objetivo de este estudio fue establecer los principales motivos para la donación voluntaria en una población que asistió a un punto de recolección de Bogotá (Colombia). Se diseñó un estudio trasversal descriptivo en una muestra de 500 adultos (28,8 $\pm 10,4$ años), $58 \%$ mujeres. La recolección de la información, se realizó aplicando una encuesta. Las variables recolectadas en el estudio fueron edad, sexo, estrato socio-económico, lugar de residencia en los últimos cinco años y principal motivo para donar sangre. Se realizó un análisis descriptivo y uno bivariado, aplicando la prueba chi2. Los principales motivos de la población donante fueron la promoción de la donación (18\% IC95\% 14,7-21,7) y salvar vidas (14,4\% IC95\% 11,4$17,8)$. Las personas de 25 años o más, donan sangre en mayor proporción por salvar vidas $(27,7 \%)$, mientras que, los menores de 25 años lo hacen principalmente como hábito saludable (26,9\%). Las personas de nivel de estrato socio-económico I y II donan motivados, principalmente, por la promoción de la donación (33,0\%), mientras que las de estratos III, IV y V lo hacen por salvar vidas $(38,4 \%)$.
La población de estudio tiene información clara sobre los aspectos que los motivan a realizar la donación voluntaria y altruista, como la promoción de la donación, salvar vidas, entre otras.

Palabras clave: Promoción, donación de sangre, estrato socio-económico, bancos de sangre.

\section{SUMMARY}

The demand for blood donation makes it a constant activity, whereby knowing the motivational aspects for its implementation becomes a determinant in the formulation of strategies for the increase of donors and to strengthen the donation programs. The aim of this study was to establish the main motivations for voluntary donation in the population that attended a gathering point of Bogotá (Colombia). A descriptive transversal study was designed with a sample of 500 adults ( $28.8 \pm 10.4$ years), $58 \%$ female. A survey was done for data collection. The variables used were age, sex, socioeconomic status, residence for the last five years and main motive for blood donating. A descriptive and a bivariate analysis applying the chi 2 test were ejecuted. The software used was Stata 11.0. The main motives for donation were the promotion (18\% CI 95\% 14.7-21.7) and to save lives (14.4\% CI 95\% 11.4-17.8). People coming from socioeconomic 
levels I and II donated mainly motivated by the promotion (33.0\%) and those from the socioeconomic levels III, IV and $\mathrm{V}$ donated mainly to save lives (38.4\%). The interviewed population gave clear information about the aspects that motivated voluntarily and willingly blood donation, as to promote more donations and save lives, among others.

Key words: Promotion, blood donation, socioeconomic level, blood bank.

\section{INTRODUCCIÓN}

La donación de sangre consiste en extraer una cantidad de 450 mililitros de sangre directamente de una vena del donante a una bolsa con anticoagulante destinada para el procesamiento de la misma. Los donantes tienen que realizar primero una entrevista de selección para descartar factores de riesgo y de antecedentes de salud; el procedimiento, según lo estipulado en el manual de normas técnicas y administrativas en banco de sangre para Colombia, determina que el periodo mínimo de tiempo establecido entre cada donación es de tres meses para hombres y cuatro meses para las mujeres, con una edad de 18 a 65 años, según lo señalado por la Organización Panamericana de Salud (1999), como población apta para donar sangre (Gallego et al. 2000).

El proceso de donación de sangre, a través del tiempo, se ha comportado como un hecho social precedido por una actitud hacia éste, en el cual, inciden todo tipo de convicciones, que influyen o motivan al sujeto a realizar la donación de una manera voluntaria y altruista (Hosain et al. 1997; Fernández, 2001). Se ha demostrado que una alta población de donantes que realizan el proceso dentro del programa de donación voluntaria y altruista está asociado con una baja tasa de resultados positivos en las pruebas realizadas, como tamizaje serológico de agentes infecciosos, situación explicada por la motivación explicita del sujeto y los aspectos propios que influyen en la realización de procedimiento; esta actitud frente a la donación permiten al momento de realizar la entrevista de selección del donante, detectar factores de riesgo y situaciones potencialmente peligrosas antes de procesamiento de la sangre (Dodd, 1989; Mundee et al. 1995; Boulware et al. 2002; Carrazzone et al. 2004).

Por lo anterior, las estrategias de captación de donantes de sangre establecidas en la actualidad por los bancos de sangre se centran en reclutar donantes voluntarios y altruistas. Los grupos de promoción de la donación determinan que una de las estrategias propuestas de abordaje comunitario es contar con información sobre el proceso de donación de sangre desde la percepción que tiene la población acerca del procedimiento.
Existen varios factores socio-culturales que influyen al momento de la donación de sangre, tales como las motivaciones frente a la realización del proceso y los diversos limitantes del mismo. Existen mitos frente a la donación, como subir o bajar de peso, el miedo a las agujas, la comercialización de los productos sanguíneos, entre otros. De esta manera, al poder conocer esta información, los grupos encargados de promocionar la donación pueden plantear estrategias eficaces que abarquen todos los aspectos psicológicos y sociales del individuo y aumentar, de esta forma, la recolección de sangre en la población (Cruz et al. 2011).

En tal sentido, antes de desarrollar una estrategia de reclutamiento de donantes de sangre, es necesario conocer los aspectos que motivan directamente al donante a realizar el procedimiento, con el propósito de orientar estrategias y alcanzar una donación voluntaria, basada en el altruismo y abandonar otras fuentes de donación, como la familia o las donaciones dirigidas a un paciente en especial que las requiere por su estado de salud, en los cuales, el acto de la donación está ligado principalmente con la necesidad (Fernández, 2001).

Se hace necesario, entonces, plantear modelos de intervención social y comunitaria, que permitan analizar las relaciones entre las variables que explican el comportamiento de los donantes y los aspectos que influyen directamente en la realización del proceso de donación voluntaria y altruista de sangre (Hupper et al. 2005; Belda et al. 2004).

Por lo anterior, el objetivo de este trabajo de investigación fue conocer los aspectos motivacionales relacionados con la donación voluntaria de sangre en una muestra de donantes repetitivos, en un banco de sangre Categoría A, de la ciudad Bogotá, Colombia.

\section{MATERIALES Y MÉTODOS}

Se realizó un estudio no experimental de tipo transversal descriptivo, en el cual, no hubo manipulación de variables ni estas requerían un control; de igual manera, se permitió describir, a partir de la aplicación de un instrumento, una encuesta, los principales aspectos motivacionales de los individuos que acceden a los servicios de donación de sangre, de la ciudad de Bogotá. La recolección de la información, se realizó en un solo periodo de tiempo, comprendido entre enero a junio de 2011, en un banco de sangre, de de Bogotá-Colombia. La selección de la muestra fue no probabilística; como criterio de inclusión, se estableció ser donante de sangre y tener entre 18 y 64 años, edad establecida por la Organización Mundial de la Salud, como población apta para donar sangre. Se excluyeron los 
individuos que no participaron voluntariamente en el estudio (Hernández, 2007).

Con el fin de validar los datos y evitar sesgos, la encuesta se diligenció mediante entrevista individual, que constaba de seis variables, descritas de la siguiente manera: edad, sexo, lugar donde vive actualmente, lugar donde vivió en los últimos cinco años, estrato socio-económico, el cual, hace referencia a una clasificación socio-económica (I al VII) y, por último, se indagó por el principal motivo de donación de sangre. Se desarrolló una prueba piloto con 20 individuos, donde se aplicó el ejercicio de sensibilización al tipo de pregunta y los ajustes pertinentes.

En el análisis descriptivo, se aplicaron medidas de tendencia central y de dispersión para las variables cuantitativas y frecuencias absolutas y relativas e intervalos de confianza del 95\% (IC95\%), para las variables cualitativas. En el análisis bivariado, se usó la prueba chi2, para comparar los principales motivos para donar sangre, según sexo, edad y estrato socioeconómico. La variable edad, se categorizó en dos grupos, usando la mediana, como punto de corte. La variable estrato socio-económico, se agrupó en dos categorías: la primera, que contiene la población de estudio con estrato socioeconómico I y II y, la segunda, incluyó los sujetos con estrato III, IV y V; no se evidenciaron sujetos con nivel de estrato VI y VIII. El nivel alpha usado fue 0,05. Previamente los datos fueron digitados en Excel versión 2007 y el procesamiento de los datos, se realizó en el programa Stata 11.0.
El desarrollo de la investigación contó con el aval de la institución y el departamento de promoción de la donación de sangre y se enmarcó en la categoría de investigación sin riesgo, según la resolución No. 008430 de 1993, del Ministerio de Salud de Colombia, que establece las normas científicas, técnicas y administrativas para la investigación en salud.

\section{RESULTADOS Y DISCUSIÓN}

En esta investigación, se observaron los principales aspectos motivacionales relacionados con la donación voluntaria de sangre de donantes repetitivos, en un banco de sangre Categoría A, de la ciudad de Bogotá, Colombia.

Características socio-demográficas: La población de estudio estuvo conformada por 500 adultos (58\% mujeres), con una edad promedio de 28,8 $\pm 10,4$ años. El $52,4 \%$ de la población tenía 25 años o más y el 63,4\% pertenecía al estrato socio-económico II (Tabla 1).

La edad descrita en el estudio tiene un comportamiento similar con estudios relacionados con población donante de sangre (Vásquez et al. 2007). En general, la población tiene aspectos positivos frente a la donación de sangre, como se evidencia en diversos estudios realizados en comunidades específicas, como universitarios de Inglaterra, de Chile y de Tailandia (Vásquez et al 2007; Wiwanikit, 2002). Estos resultados son esperados, teniendo en cuenta la cultura de donación que se ha impulsado en el ámbito universitario,

Tabla 1. Características socio-demográficas de la población de estudio.

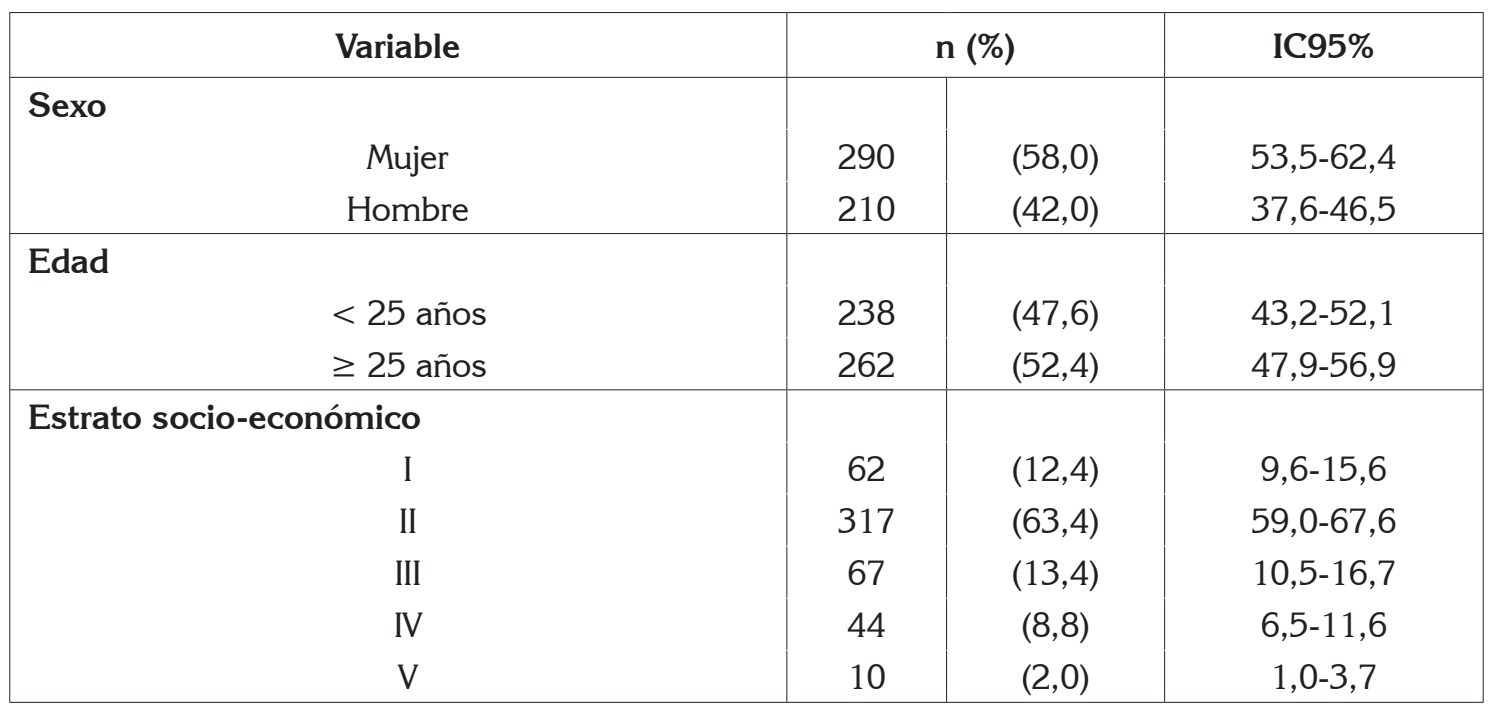


creando conciencia en las poblaciones jóvenes. En cuanto al nivel de estrato socio-económico de la población, la disposición de tiempo es un factor determinante, para acceder al proceso de donación.

La población de estudio reside al momento de la encuesta, principalmente, en las siguientes localidades, de la ciudad de Bogotá: Suba $(12,0 \%)$ y Kennedy $(11,2 \%)$ como los datos más significativos (Figura 1) y en los últimos cinco años, el
76,6\% de los participantes refirió vivir en la ciudad de Bogotá, mientras que el resto de la población residió en otros lugares de Colombia (Figura 2). De acuerdo al comportamiento de la información suministrada por la población en relación a la tendencia en la participación, según la ubicación geográfica en los procesos de donación, la procedencia no genera información de relevancia para generalizar sobre la población de donantes de la ciudad de Bogotá, pero sí se comporta similar a un estudio realizado en esta ciudad (Cruz et al. 2011).

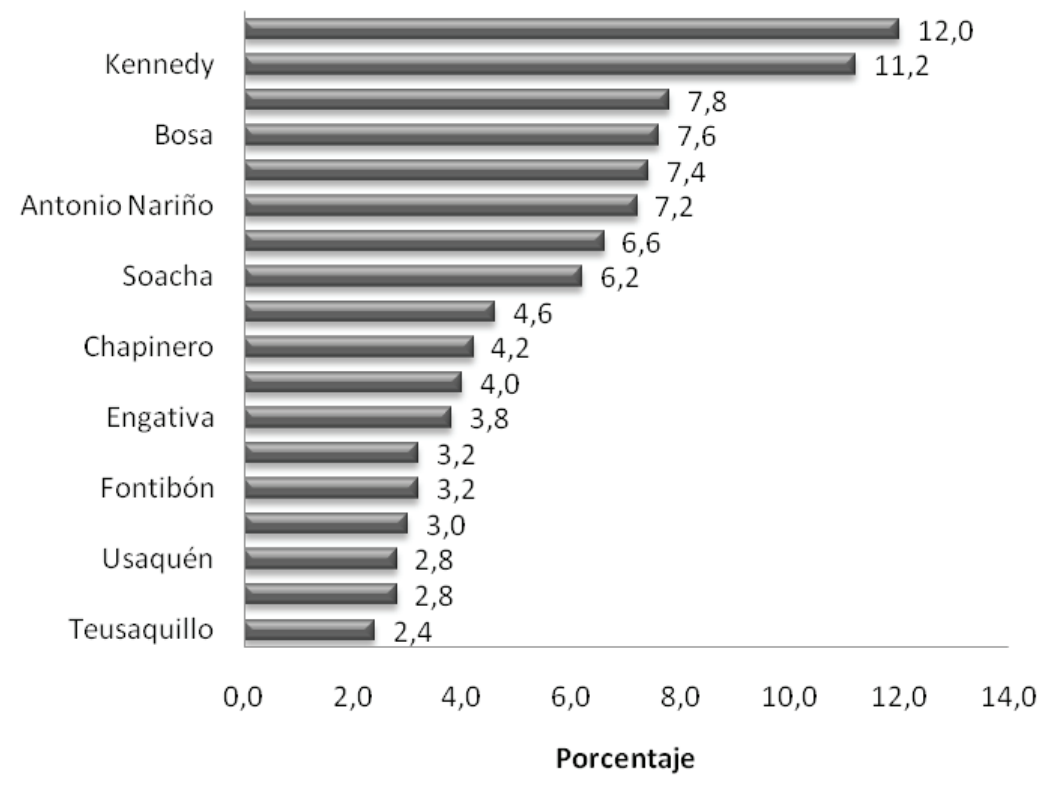

Figura 1. Lugar donde vive actualmente la población de estudio, 2011.

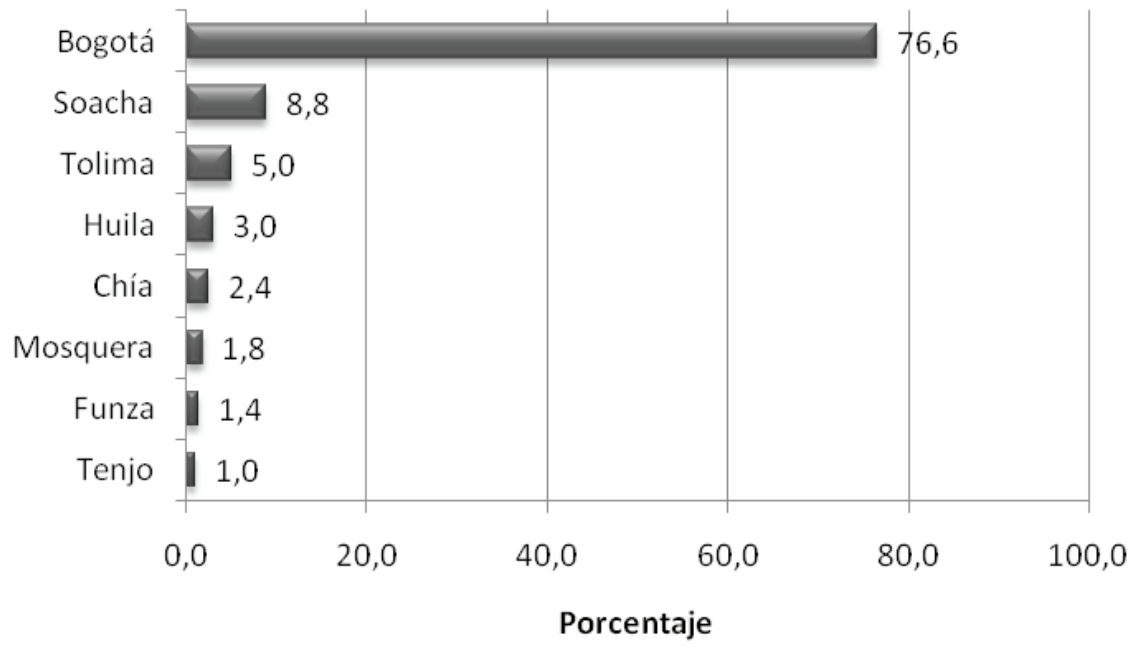

Figura 2. Lugar de residencia en los últimos cinco años de la población de estudio. 
Motivaciones frente a la donación de sangre: Dentro de los motivos para donar sangre, se resaltan las jornadas de promoción de la donación, con el $18 \%$; salvar vidas, con el $14,4 \%$ y considerar que es un hábito saludable, con el 13,8\% (Tabla 2). Las campañas de promoción de la donación deben tener en cuenta los aspectos motivacionales encontrados, según el sexo. En relación a lo anterior, diversos estudios establecen que, en la actualidad, los hombres y las mujeres identifican claramente aspectos en común frente a la donación voluntaria y altruista de sangre (Fernández. 2001; Vásquez et al. 2007; Boulware et al. 2002; Buciuniene et al. 2006). En relación a los aspectos contemplados en promoción de la donación, la población establece claramente que ésta es la principal motivación que los direcciona hacia la realización de la donación de sangre de manera voluntaria y altruista, por lo cual, la información proporcionada por este estudio puede llegar a ser útil, en la medida que los bancos y los grupos encargados de promoción de la donación y de mercadeo social fortalezcan los mecanismos de abordaje a los potenciales donantes de sangre (García et al. 2003; Beltrán \& Guzmán, 2002). En este sentido, se recomienda articular las campañas de donación de sangre con los motivos para la donación de la misma (Moore, 1991; Marantidou et al. 2007). Por experiencia de nuestro grupo de promoción de la donación, se ha trabajado en las necesidades de los donantes potenciales, buscando abordarlos desde la forma en que ellos desean que las campañas promocionales los aborden en este tipo de temáticas.

Tabla 2. Principales motivos para donar sangre en la población de estudio, 2011.

\begin{tabular}{|l|c|c|c|}
\hline Motivo para donar sangre & $\mathbf{n}$ & $\mathbf{\%}$ & $\mathbf{I C 9 5 \%}$ \\
\hline Promoción de la donación & 90 & $(18,0)$ & $14,7-21,7$ \\
\hline Salvar vidas & 72 & $(14,4)$ & $11,4-17,8$ \\
\hline Hábito saludable & 69 & $(13,8)$ & $10,9-17,1$ \\
\hline Curiosidad ante el proceso & 66 & $(13,2)$ & $10,4-16,5$ \\
\hline Tipo de sangre es escaso & 47 & $(9,4)$ & $7,0-12,3$ \\
\hline Compromiso social & 33 & $(6,6)$ & $4,6-9,1$ \\
\hline Invitación a donar diferente a promoción de la donación & 33 & $(6,6)$ & $4,6-9,1$ \\
\hline Estilo de vida & 31 & $(6,2)$ & $4,3-8,7$ \\
\hline Voluntad de donar, donante frecuente & 26 & $(5,2)$ & $3,4-7,5$ \\
\hline Generosidad & 25 & $(5,0)$ & $3,3-7,3$ \\
\hline Colaboración, ayuda y cultura & 8 & $(1,6)$ & $0,7-3,1$ \\
\hline
\end{tabular}

Por otra parte, se encontró que los motivos para donar sangre no son estadísticamente diferentes entre hombres y mujeres, como se evidenciaba anteriormente, en donde las mujeres eran quienes acudían principalmente a los procesos de donación de sangre. En contraste, se observaron diferencias estadísticamente por edad $(p=0,001)$. Las personas de 25 años o más donan sangre en mayor proporción por salvar vidas, con un $27,7 \%$, comparado con los menores de 25 años, en donde esta motivación es del $13,1 \%$, mientras que los menores de 25 años, lo hacen, principalmente, como hábito saludable, con un $26,9 \%$, comparado con el $14,1 \%$, de las mayores de 25 años (Tabla 3). En general, los jóvenes se encuentran generando nuevos estilos de vida, entre los que encontramos los hábitos relacionados con el cuidado de la salud; en general, los jóvenes se constituyen hoy en una población que hay que explorar en temas relacionados con donación de sangre.

Así mismo, se observaron diferencias estadísticamente significativas por estrato socio-económico $(p=0,000)$. Las personas de nivel de estrato I y II donan motivados, principalmente, por la promoción de la donación, con un $33,0 \%$, a diferencia de los estratos III, IV y V, en donde esta motivación es del 5,8\%. Por el contrario, las personas de estratos III, IV y V donan, principalmente, por salvar vidas, con un $38,4 \%$, comparado con el $15,1 \%$, de las personas de estratos I y II (Tabla 3). Estos resultado son esperados para nuestros objetivos de promoción de la donación, en los cuales, los procesos educativos van encaminados hacia poblaciones que no conocen sobre el proceso de la donación.

La población encuestada señaló aspectos sociales, como salvar vidas, como una de las principales motivaciones hacia la donación, situación enmarcada en varios estudios relacionados con banco de sangre (Cruz et al. 2011; Hupper et al. 2005; Vásquez et al. 2007; Wiwanikit, 2002). De la misma manera, el altruismo que genera la donación de 
Tabla 3. Principales motivos para donar sangre en la población de estudio según sexo, edad y estrato socio-económico.

\begin{tabular}{|c|c|c|c|c|c|c|c|c|}
\hline \multirow[b]{2}{*}{ Variabl } & & \multicolumn{5}{|c|}{ Motivos para donar sangre en los participantes } & \multirow[b]{2}{*}{ Subtotal } & \multirow{2}{*}{$\begin{array}{c}\text { Valor } \\
\mathrm{p}^{*}\end{array}$} \\
\hline & & $\begin{array}{l}\text { Promoción de } \\
\text { la donación }\end{array}$ & $\begin{array}{l}\text { Salvar } \\
\text { vidas }\end{array}$ & $\begin{array}{c}\text { Hábito } \\
\text { saludable }\end{array}$ & $\begin{array}{l}\text { Curiosidad al } \\
\text { Proceso }\end{array}$ & $\begin{array}{c}\text { Tipo de } \\
\text { Sangre Escaso }\end{array}$ & & \\
\hline \multicolumn{8}{|l|}{ Sexo } & \multirow{5}{*}{0,263} \\
\hline \multirow{4}{*}{ Femenino } & $\mathrm{n}$ & 43 & 23 & 27 & 30 & 17 & 140 & \\
\hline & $\%$ & $(30,7)$ & $(16,4)$ & $(19,3)$ & $(21,4)$ & $(12,2)$ & 100,0 & \\
\hline & $\mathrm{n}$ & 47 & 49 & 42 & 36 & 30 & 204 & \\
\hline & $\%$ & $(23,0)$ & $(24,0)$ & $(20,6)$ & $(17,7)$ & $(14,7)$ & 100,0 & \\
\hline \multicolumn{8}{|l|}{ Edad } & \multirow{5}{*}{0,001} \\
\hline \multirow{4}{*}{$\geq 25$ años } & $\mathrm{n}$ & 41 & 21 & 43 & 36 & 19 & 160 & \\
\hline & $\%$ & $(25,6)$ & $(13,1)$ & $(26,9)$ & $(22,5)$ & $(11,9)$ & 100,0 & \\
\hline & $\mathrm{n}$ & 49 & 51 & 26 & 30 & 28 & 184 & \\
\hline & $\%$ & $(26,7)$ & $(27,7)$ & $(14,1)$ & $(16,3)$ & $(15,2)$ & 100,0 & \\
\hline \multicolumn{2}{|c|}{\begin{tabular}{|l|} 
Estrato \\
socio-económico
\end{tabular}} & & & & & & & \multirow{5}{*}{0,000} \\
\hline I y II & n & 85 & 39 & 46 & 50 & 38 & 258 & \\
\hline 1011 & $\%$ & $(33,0)$ & $(15,1)$ & $(17,8)$ & $(19,4)$ & $(14,7)$ & 100,0 & \\
\hline \multirow{2}{*}{ III, IV y V } & $\mathrm{n}$ & 5 & 33 & 23 & 16 & 9 & 86 & \\
\hline & $\%$ & $(5,8)$ & $(38,4)$ & $(26,7)$ & $(18,6)$ & $(10,5)$ & 100,0 & \\
\hline
\end{tabular}

*Prueba Chi2

sangre ha sido un tema bastante estudiado en la adopción de conductas sociales deseadas en la población (Fernández, 2001; Zaller \& Nelson, 2005; Misje et al. 2005; Marantidou et al. 2007).

En general, los participantes en el estudio informan las motivaciones para realizar el proceso de donación de sangre, aunque es difícil obtener participación de personas con algunos grupos sanguíneos. Fue evidente una notable conciencia social frente a la necesidad de la población, puesto que la sangre es un recurso vital. De igual manera, la curiosidad ante el procedimiento y el tipo de sangre escaso, como un motivo para la donación, se puede ver influenciada por conocimientos previos acerca de la necesidad de sangre en la actualidad (Beltrán \& Guzmán, 2002).

Este estudio aportó información acerca de aspectos que motivan a realizar la donación de sangre; así mismo recolectó y analizó información sobre el tema, que permite al departamento de promoción de la donación de la Fundación Hematológica Colombia y demás bancos de sangre interesados en el tema propuesto para la información y comunidad en general, contar con un acercamiento frente a información proporcionada por la comunidad. Una de las limitantes del estudio es no poder hacer una generalización de los hallazgos, por las características del diseño y el tipo de selección de la muestra, la cual, es de tipo no probabilística, con una selección por conveniencia y no representa a toda la población de donantes de Colombia.

Este estudio genera como conclusiones, que la población objeto tiene información clara sobre los aspectos que los motivan a realizar la donación voluntaria y altruista de sangre; de la misma manera, se establece que no hay diferencia entre donantes de sangre por sexo, como se habla en estudios previos respecto a este tema. Información que cambia la perspectiva que se tenía en relación a los donantes de sangre, donde se consideraba que las mujeres eran quienes, principalmente, accedían a los puntos de recolección de sangre.

La mayoría de la población realiza la donación basada en procesos de educación en salud brindados por la promoción de la donación, pero también son importantes los aspectos propios del ser humano, los cuales, no se ven direccionados por este tipo de procesos de marketing social. Se requiere realizar investigaciones en otro tipo de población, para construir datos significativos, acerca del tema. Las 
investigaciones en Colombia sobre donación de sangre han sido centradas, fundamentalmente, sobre las causas, por las cuales, no se ejecuta esta práctica y no se ha indagado sobre los aspectos motivacionales frente a un proceso tan difícil de abordar, a través de la historia de la promoción de la donación. Se recomienda a los investigadores, tomar una muestra más representativa, que incluya diferentes ciudades de Colombia, agregando otras variables, como miedos frente a la donación, con el fin de establecer integralmente las actitudes, tanto positivas como negativas en la población. Por otra parte, se recomienda hacer estudios únicamente en población joven y, en especial, universitaria, para conocer cuáles, específicamente, son las motivaciones de esta población.

Agradecimientos: Fundación Hematológica Colombia y al departamento de promoción de la donación de sangre. Al grupo de auxiliares de enfermería y enfermeros de las campañas de recolección de sangre por su colaboración en la recolección de datos. Conflictos de interés: Este artículo fue preparado y revisado con la participación de todos los autores, quienes declaran que no existe ningún conflicto de intereses que ponga en riesgo la validez de los resultados.

\section{BIBLIOGRAFÍA}

1. BELDA, I.; FERNANDEZ, A.; RODRIGUEZ, A.; LOPEZBERRIO, A. 2004. How regular blood donors explain their behavior. Transfusión. 44:1441-1446.

2. BELTRÁN, M.; GUZMÁN, N. 2002. Conocimientos, actitudes y motivaciones sobre la donación de sangre en la comunidad universitaria de Bogotá, Colombia. Med. Transf. al Día. 2(3):168-171.

3. BOULWARE, L.; RATNER, L.; NESS, P.; COOPER, L.; CAMPBELL-LEE, S.; LAVEIST, T. 2002. The contribution of sociodemographic, medical, and attitudinal factors to blood donation among the general public. Transfusión. 42:669-678.

4. BUCIUNIENE, I.; STONIENË, L.; BLAZEVICIENE, A.; KAZLAUSKAITE, R.; SKUDIENE, V. 2006. Blood donors' motivation and attitude to non-remunerated blood donation in Lithuania. BMC Public Health 6:166.

5. CRUZ, H.; MORENO, J.; ANGARITA, A.; CALDERÓN, C.; MARTÍNEZ, S.; RESTREPO, M. 2011. Imaginarios sociales de donantes Voluntarios de sangre en un punto fijo de recolección. Bogotá, Colombia. Rev. Invest. Andina. 23(13):250-257.
6. CARRAZZONE, C.F.V.; BRITO, A.M.; GOMES, Y.M. 2004. Importância da avaliação sorológica prétransfusional em receptores de sangue. Rev. Bras. Hematol. Hemoterapia. 26(2):93-98.

7. DODD, R.Y. 1989. Screening for hepatitis infectivity among blood donors: a model for blood safety. Arch. Path. Lab. Med. 113(3):227-231.

8. FERNÁNDEZ, A. 2001. La donación de sangre voluntaria y altruista: Un reto irrenunciable. SETS. 40:1-2.

9. GALlEGO, M.; MUÑOZ, L.; CORTÉS, A. 2000. Características socioculturales de los donantes y no donantes de sangre en Colombia. Colombia Méd. 31:99-109.

10. GARCÍA, G.; SÁENZ, E.; CRUZ, R. 2003. Estudio de factores socioculturales relacionados con la donación voluntaria de sangre en las Américas. Rev. Panam. Salud PúbL. 13(2-3):85-90.

11. HERNÁNDEZ, M. 2007. Epidemiología, diseño y análisis de estudios. Instituto Nacional de Salud Pública. Editorial Médica Panamericana (Colombia). 382p.

12. HOSAIN, G.M.; ANISUZZAMAN, M.; BEGUR, A. 1997. Knowledge and attitude towards voluntary blood donation among Dhaka University students in Bangladesh. East Afr. Med. J. 74(9):549-53.

13. HUPPER, M.; TAYLOR, D.; LETWIN, J. 2005. Understanding canadian student motivations and beliefs about giving blood. Transfusion. 45:149-161.

14. MARANTIDOU, O.; LOUKOPOULOU, L.; ZERVOU, E.; MARTINIS, G.; EGGLEZOU, A.; FOUNTOULI, P.; DIMOXENOUS, P.; PARARA, M.; GAVALAKI, M.; MANIATIS, A. 2007. Factors that motivate and hinder blood donation in Greece. Transf. Med. 17:443-450.

15. MISJE, A.; BOSNES, V.; GASDAL, O. 2005. Motivation, recruitment and retention of voluntary nonremunerated blood donors: a survey-based questionnaire study. Vox Sanguinis. 89(4):236-244.

16. MOORE, R. 1991. Promoting blood donation: a study of the social profile, attitudes, motivations and experience of donors. Transf. Med. 1(4):201-207.

17. MUNDEE, Y.; KAMTORN, N.; CHAIYAPHRUK, S.; NANTACHIT, N.; NESS, P.M.; NELSON, K.E. 1995. 
Infectious disease markers in blood donors in northern Thailand. Transfusion. 3:264-267.

18. ORGANIZACIÓN PANAMERICANA DE LA SALUD. 1999. División de Desarrollo de Sistemas y Servicios de Salud. Guía metodológica para investigación de aspectos socio-culturales relacionados con la donación voluntaria de sangre. Washington D.C.: Organización Panamericana de la Salud. Serie Medicamentos Esenciales y Tecnología. 11p.

19. VÁSQUEZ, M.; IBARRA, P.; MALDONADO, M. 2007. Conocimientos y actitudes hacia la donación de sangre en una población universitaria de Chile. Rev. Panam. Salud Públ. 22(5):323-328.

20. WIWANIKIT, V. 2002. Knowledge about blood donation among a sample of Thai university students. Vox Sanguinis. 83(2):97-99.

21. ZALLER, N.; NELSON, K. 2005. Knowledge, attitude and practice survey regarding blood donation in a Northwestern Chinese city. Transf. Med. 15:277-286.

Recibido: Noviembre 3 de 2011

Aceptado: Abril 25 de 2012 